\title{
On the dispersionless Kadomtsev-Petviashvili equation in $n+1$ dimensions: exact solutions, the Cauchy problem for small initial data and wave breaking
}

\author{
S. V. Manakov ${ }^{1, \S}$ and P. M. Santini ${ }^{2, \S}$ \\ ${ }^{1}$ Landau Institute for Theoretical Physics, Moscow, Russia \\ 2 Dipartimento di Fisica, Università di Roma "La Sapienza", and \\ Istituto Nazionale di Fisica Nucleare, Sezione di Roma 1 \\ Piazz.le Aldo Moro 2, I-00185 Roma, Italy

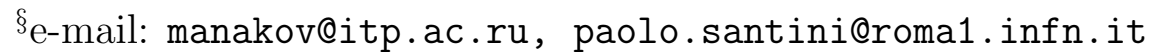

April 22, 2022

\begin{abstract}
We study the $n+1$-dimensional generalization of the dispersionless Kadomtsev-Petviashvili (dKP) equation, a universal equation describing the propagation of weakly nonlinear, quasi one dimensional waves in $n+1$ dimensions, and arising in several physical contexts, like acoustics, plasma physics and hydrodynamics. For $n=2$, this equation is integrable, and it has been recently shown to be a prototype model equation in the description of the two dimensional wave breaking of localized initial data. We construct an exact solution of the $n+1$ dimensional model containing an arbitrary function of one variable, corresponding to its parabolic invariance, describing waves, constant on their paraboloidal wave front, breaking simultaneously in all points of it. Then we use such solution to build a uniform approximation of the solution of the Cauchy problem, for small and localized initial data, showing that such a small and localized initial data evolving according to the $n+1$-dimensional dKP equation break, in the long time regime, if and only if $1 \leq n \leq 3$; i.e., in physical space. Such a wave breaking takes place, generically, in a point of the paraboloidal wave front, and the analytic aspects of it are given explicitly in terms of the small initial data.
\end{abstract}




\section{Introduction}

The $n+1$ dimensional generalization of the dispersionless Kadomtsev - Petviashvili equation:

$$
\begin{aligned}
& \left(u_{t}+u u_{x}\right)_{x}+\Delta_{\perp} u=0, \quad u=u(x, \vec{y}, t), \quad \vec{y}=\left(y_{1}, \ldots, y_{n-1}\right) \\
& \Delta_{\perp}=\sum_{i=1}^{n-1} \partial_{y_{i}}^{2}, \quad n \geq 2
\end{aligned}
$$

hereafter referred to as the $d K P_{n}$ equation, describes the propagation of weakly nonlinear quasi one dimensional waves in $n+1$ dimensions. This equation was first obtained by Timman [1], for $n=2$, in the study of unsteady motion in transonic flows, and then derived by Khoklov - Zobolotskaya, in the $3+1$ dimensional acoustic context in [2], where the main properties of the model were pointed out. Equation (1) is the $x$-dispersionless limit of another distinguished model, the $n+1$ dimensional generalization of the Kadomtsev-Petviashvili equation [3], integrable for $n=2$ by the Inverse Spectral Transform (IST) [4, 5].

The universal character of (11) can be explained as follows. Take any system of nonlinear PDEs i) characterized, for example, by nonlinearities of hydrodynamic type and ii) whose linear limit, at least in some approximation, is described by the wave equation. Then, iii) looking at the propagation of quasi - one dimensional waves and iv) neglecting dispersion and dissipation, one obtains, at the second order in the proper multiscale expansion, the $d K P_{n}$ equation (11). Therefore (1) arises in several physical contexts, like acoustics, plasma physics and hydrodynamics.

Indeed, consider a nonlinear system of PDEs whose linear limit is the wave equation $f_{t^{\prime} t^{\prime}}=\Delta f$ in $n+1$ dimensions; then a localized initial condition evolving according to it is concentrated, asymptotically, on the spherical wave front:

$$
|\vec{x}|-t^{\prime}=O(1), \quad|\vec{x}|=\sqrt{\sum_{k=1}^{n} x_{k}^{2}} .
$$

Looking for nonlinear corrections and quasi - one dimensional propagations (say, in the $x_{1}$ direction), we introduce the convenient variables:

$$
x=x_{1}-t^{\prime}=O(1), \quad y_{j}=\epsilon^{q} x_{j+1}, j=1, \ldots, n-1, \quad t=\epsilon^{2 q} t^{\prime}, \quad q>0,
$$

where $\epsilon$ is the order of magnitude of the small amplitude of the wave. Then the spherical wave front becomes, approximately, its second order contact, 
an ellipsoidal paraboloid:

$$
|\vec{x}|-t^{\prime} \sim x+\frac{1}{2 t} \sum_{k=1}^{n-1} y_{k}^{2}=O(1) .
$$

In addition, starting from the dispersion relation of the wave equation and looking for quasi one dimensional waves in the $x_{1}$ direction, we have longer wave lengths (smaller wave numbers) in the transversal directions: $\vec{k}_{\perp}=$ $\epsilon^{q} \vec{\kappa}_{\perp}$, obtaining that the dispersion relation $\omega(\vec{k})$ of the wave equation reduces to that of the linearized $d K P_{n}$ (11) (up to the trivial rescaling $\vec{y} \rightarrow \vec{y} / \sqrt{2}$ ):

$$
\begin{aligned}
& \omega(\vec{k})=\sqrt{\sum_{j=1}^{n} k_{j}^{2}} \sim k_{1}+\epsilon^{2 q} \frac{\kappa_{\perp}^{2}}{2 k_{1}}, \quad \kappa_{\perp}^{2}=\sum_{j=2}^{n} \kappa_{j}^{2}, \\
& \theta\left(\vec{x}, t^{\prime}\right)=\vec{k} \cdot \vec{x}-\omega(\vec{k}) t^{\prime} \sim k_{1} x+\vec{\kappa}_{\perp} \cdot \vec{y}-\frac{\kappa_{\perp}^{2}}{2 k_{1}} t .
\end{aligned}
$$

This is what happens in the acoustic problem in $3+1$ dimensions [2], where the gas density, the pressure and the component of the velocity in the main direction $x_{1}$ are proportional to $\epsilon u(x, \vec{y}, t)$, and $u$ solves the $d K P_{3}$ equation.

Starting from the plasma physics equations

$$
\rho_{t}+\nabla \cdot(\rho \vec{v})=0, \quad \vec{v}_{t}+(\vec{v} \cdot \nabla) \vec{v}+\nabla \phi=\overrightarrow{0}, \quad \Delta \phi-e^{\phi}+\rho=0,
$$

describing a gas of hot electrons on a background of cold ions, where $\rho$ and $\vec{v}$ are the ion density and velocity and $\phi$ is the electric potential [6], the linearized theory leads instead to a generalized fourth order wave equation in $(3+1)$ dimensions

$$
\Delta \phi_{t t}=\phi_{t t}-\Delta \phi
$$

reducing to the wave equation in the long wave approximation (small wave numbers):

$$
\omega=\sqrt{\frac{k^{2}}{1+k^{2}}} \sim k\left(1-\frac{k^{2}}{2}\right), \quad k^{2}=k_{1}^{2}+k_{2}^{2}+k_{3}^{2} .
$$

Looking, in addition, for quasi one dimensional waves (the wave numbers in the transversal directions are smaller than the (small) wave number in the 
$x_{1}$ direction: $\left.k_{1}=\epsilon^{p} \kappa_{1}, \vec{k}_{\perp}=\epsilon^{p+q} \vec{\kappa}_{\perp}\right)$, we obtain

$$
\begin{aligned}
& \omega=\sqrt{\frac{k^{2}}{1+k^{2}}} \sim \epsilon^{p} \kappa_{1}+\epsilon^{p+2 q} \frac{\kappa_{\perp}^{2}}{2 \kappa_{1}}-\epsilon^{3 p} \frac{\kappa_{1}^{3}}{2}, \\
& \theta\left(\vec{x}, t^{\prime}\right)=\vec{k} \cdot \vec{x}-\omega(\vec{k}) t^{\prime} \sim \\
& \kappa_{1} x+\vec{\kappa}_{\perp} \cdot \vec{y}-\left\{\begin{array}{cc}
\epsilon^{p+2 q} \frac{\kappa_{\perp}^{2}}{2 \kappa_{1}} t, & p>2 q, \\
\epsilon^{3 q}\left(\frac{\kappa_{\perp}^{2}}{2 \kappa_{1}}-\frac{\kappa_{1}^{3}}{2}\right) t, & p=2 q,
\end{array}\right.
\end{aligned}
$$

where now

$$
x=\epsilon^{p}\left(x_{1}-t^{\prime}\right), \quad y_{j}=\epsilon^{p+q} x_{j+1}, \quad j=1,2, \quad t=\epsilon^{p+2 q} t^{\prime} .
$$

If $p>2 q$, the term $k_{1}^{3}$ is negligeable wrt $\left(\kappa_{\perp}^{2}\right) / \kappa_{1}$ and one obtains again the dispersion relation of the linearized $d K P_{3}$ equation; if $p=2 q$, they are comparable and one obtains the dispersion relation of the linearized KP equation in $3+1$ dimensions.

In the water wave theory, the situation is very similar to that of plasma physics and the $d K P_{2}$ equation is derived in the long wave approximation. In all the above three physical contexts, the choice of the exponent $q$ in (3), (10) comes from the balance, at the second order in the proper multiscale expansion, with the nonlinearity of the physical system, and is $q=1 / 2$.

We remark that the $1+1$ dimensional version of (1) is the celebrated Riemann-Hopf equation $u_{t}+u u_{x}=0$, the prototype model in the description of the gradient catastrophe (or wave breaking) of one dimensional waves [7]. Therefore a natural question arises: do solutions of $d K P_{n}$ break and, if so, is it possible to give an analytic description of such a multidimensional wave breaking?

A first and positive answer in this direction was recently given in [8], for the integrable [9, 10, 11, 12, 13, 14] $d K P_{2}$ case. Indeed, using a novel IST for vector fields, we have been able to solve the Cauchy problem of $d K P_{2}$ [15] and of other distinguished integrable Partial Differential Equations (PDEs) arising as commutation of multidimensional vector fields [16, 17, 18]. The associated nonlinear Riemann-Hilbert (RH) inverse problem turns out to be an efficient tool to study several properties of the solution space of $d K P_{2}$, like, for instance, i) the construction of the longtime behaviour of the solutions of the Cauchy problem; ii) the possibility of establishing that localized initial profiles evolving according to $\mathrm{dKP}_{2}$ break at finite time and, if small, they break in the longtime regime, investigating in an explicit way the analytic aspects of such a longtime wave breaking of two-dimensional waves [8]. 
In this paper, motivated by the analytic results of the integrable case $n=2$ [8], we answer the above question in arbitrary dimensions, when the model (11) is not integrable, under the assumption of small and localized initial data. To obtain this result, we first construct an exact solution of equation (11) containing an arbitrary function of one variable, consequence of the parabolic invariance of equation (11), describing a wave, constant on its paraboloidal wave front, breaking simultaneously in all points of it. Then we use such solution to build a uniform approximation of the solution of the Cauchy problem, showing that "small and localized initial data evolving according to the $d K P_{n}$ equation break, in the long time regime, if and only if $1 \leq n \leq 3$; i.e., in physical space". Such a wave breaking takes place, generically, in a point of the paraboloidal wave front, and the analytic aspects of it are given explicitly in terms of the initial data. In addition we show that, if the initial data are $O(\epsilon)$, then the breaking times are respectively $O\left(\epsilon^{-1}\right), O\left(\epsilon^{-2}\right)$ and

$O\left(e^{\epsilon^{-1}}\right)$, for $n=1,2$ and 3. We remark that, from the knowledge of such a breaking longtime regime of the solution, one can reconstruct exactly the initial data, an important issue in many physical contexts.

The existence of a critical dimensionality above which small and localized data do not break has a clear origin, since, in the model, two terms act in opposite way: the nonlinearity is responsible for the steepening of the profile, while the $n-1$ diffraction channels, represented by the transversal Laplacian, have an opposite effect; for $n=1,2,3$ the nonlinearity prevails and wave breaking takes place (but at longer and longer time scales, as $n$ increases), while, for $n \geq 4$, the number of transversal diffraction channels is enough to prevent such phenomenon, for small data, in the longtime regime.

The paper is organized as follows. In $\S 2$ we derive the exact solutions of the model and in $\S 3$ we use them to build a uniform approximation of the solution of the Cauchy problem of (1), for small and localized initial data, establishing that wave breaking takes place only if $n=1,2,3$. At last, in $\S 4$, we discuss in great detail the analytic aspects of such a wave breaking.

\section{Exact solutions of the $d K P_{n}$ equation}

The universal properties of the $d K P_{n}$ equation, discussed in the Introduction, suggest its invariance under motions on the associated paraboloid. Indeed, it is easy to show that equation (11) admits the following Lie point symmetry 
group of transformations

$$
\begin{aligned}
& \tilde{x}=x+\sum_{i=1}^{n-1}\left(\delta_{i} y_{i}-\delta_{i}^{2} t\right), \\
& \tilde{y}_{j}=y_{j}-2 \delta_{j} t, \quad j=1, \ldots, n-1,
\end{aligned}
$$

where the $\delta_{j}$ 's are the arbitrary parameters of the group, leaving invariant the paraboloid

$$
x+\frac{1}{4 t} \sum_{i=1}^{n-1} y_{i}^{2}=\xi .
$$

Correspondingly, equation (11) possesses the following exact solution

$$
u= \begin{cases}t^{-\frac{n-1}{2}} F\left(x+\frac{1}{4 t} \sum_{i=1}^{n-1} y_{i}^{2}-\frac{2 u t}{3-n}\right), & n \neq 3, \\ t^{-1} F\left(x+\frac{1}{4 t} \sum_{i=1}^{n-1} y_{i}^{2}-u t \ln t\right), & n=3,\end{cases}
$$

where $F$ is an arbitrary function of one argument; such solution is characterized by the differential constraint $\sigma=0$, where $\sigma$ is the corresponding "characteristic symmetry"

$$
\sigma=\left(\sum_{i=1}^{n-1} \delta_{i} y_{i}\right) u_{x}-2 t \sum_{i=1}^{n-1} \delta_{i} u_{y_{i}}
$$

of equation (11). Indeed, if one looks for solutions of (1) in the form

$$
u=v(\xi, t), \quad \xi=x+\frac{1}{4 t} \sum_{i=1}^{n-1} y_{i}^{2}
$$

one obtains the following equation for $v(\xi, t)$ :

$$
v_{t}+\frac{n-1}{2 t} v+v v_{\xi}=0 .
$$

Its $v / t$ term can be eliminated by the change of variables

$$
v(\xi, t)=t^{-\frac{n-1}{2}} q(\xi, \tau),
$$

where

$$
\tau(t)= \begin{cases}\frac{2}{3-n} t^{\frac{3-n}{2}}, & n \neq 3 \\ \ln t, & n=3\end{cases}
$$


leading to the Riemann - Hopf equation:

$$
q_{\tau}+q q_{\xi}=0,
$$

whose general solution is implicitely given by

$$
q=F(\xi-q \tau),
$$

where $F$ is an arbitrary function of one argument. Going back to the original variables via (15), (17), (18), the solution (20) becomes (13).

We remark that, if $F$ is a regular and localized function of its argument, the solution (13) describes a wave concentrated on the wave front, given by the paraboloid (12), and constant on it, breaking, simultaneously, on the whole paraboloid. We also remark that, for $n=2$, the solution (13) has been first derived using a recently developed method to construct exact solutions of the nonlinear $\mathrm{RH}$ inverse problem associated with the integrable $d K \mathrm{P}_{2}$ equation [19].

\section{The Cauchy problem and wave breaking of small and localized initial data}

Since the paraboloid (12) plays an important role in the asymptotics of the $d K P_{n}$ equation (see the Introduction), the exact solution (13) is physically relevant and can be used to build a uniform approximation of the solution of the Cauchy problem for the $d K P_{n}$ equation, under the hypothesis of small and localized initial data.

The basic idea (already used in [20], in a different context) is that, if the initial condition is small:

$$
u(x, \vec{y}, 0)=\epsilon u_{0}(x, \vec{y}), \quad 0<\epsilon<<1,
$$

the solution of the Cauchy problem for $d K P_{n}$ is well approximated by the corresponding solution for the linearized $d K P_{n}$ untill one enters the nonlinear regime, in which the Riemann - Hopf equation (19) becomes relevant, eventually causing wave breaking. Since the breaking time of $O(\epsilon)$ initial data evolving according to (19) is $\tau(t)=O\left(\epsilon^{-1}\right)$, the nonlinear regime for $d K P_{n}$ is characterized by the condition $t=O\left(\tau^{-1}\left(\epsilon^{-1}\right)\right)$, where $\tau^{-1}$ is the 
inverse of (18); so that:

$$
\tau^{-1}\left(\epsilon^{-1}\right)=\left\{\begin{array}{cc}
\epsilon^{-\frac{2}{3-n}} & \text { if } 1 \leq n<3 \\
e^{\epsilon^{-1}} & \text { if } n=3
\end{array}\right.
$$

and a proper matching has to be made between the solution of the linearized $d K P_{n}$, valid for $t<<O\left(\tau^{-1}\left(\epsilon^{-1}\right)\right)$, and the exact solution of the previous section, valid in the nonlinear regime $t=O\left(\tau^{-1}\left(\epsilon^{-1}\right)\right)$.

\subsection{The linear regime}

Since the initial condition (21) is small, the solution of $d K P_{n}$ is well approximated, for finite times, by the solution of the linearized $d K P_{n}$ equation:

$$
u(x, \vec{y}, t) \sim \frac{\epsilon}{(2 \pi)^{n}} \int_{\mathbb{R}^{n}} \hat{u}_{0}\left(k_{1}, \vec{k}_{\perp}\right) e^{i\left(k_{1} x+\vec{k}_{\perp} \cdot \vec{y}-\frac{k_{\perp}^{2}}{k_{1}} t\right)} d k_{1} d \vec{k}_{\perp}
$$

where $\hat{u}_{0}\left(k_{1}, \vec{k}_{\perp}\right)$ is the Fourier transform of $u_{0}(x, \vec{y})$

$$
\hat{u}_{0}\left(k_{1}, \vec{k}_{\perp}\right)=\int_{\mathbb{R}^{n}} u_{0}(x, \vec{y}) e^{-i\left(k_{1} x+\vec{k}_{\perp} \cdot \vec{y}\right)} d x d \vec{y} .
$$

Such approximation is also valid in the longtime interval:

$$
1<<t<O\left(\tau^{-1}\left(\epsilon^{-1}\right)\right)
$$

(far away from the nonlinear regime), in which the solution of $d K P_{n}$ is described by the standard stationary phase approximation of the multiple integral (23):

$$
u(x, \vec{y}, t) \sim t^{-\frac{n-1}{2}} \epsilon G\left(x+\frac{1}{4 t} \sum_{i=1}^{n-1} y_{i}^{2}, \frac{\vec{y}}{2 t}\right)
$$

where

$$
G(\xi, \vec{\eta}):=2^{-n} \pi^{-\frac{n+1}{2}} \int_{\mathbb{R}} d \lambda|\lambda|^{\frac{n-1}{2}} \hat{u}_{0}(\lambda, \lambda \vec{\eta}) e^{i \lambda \xi-i \frac{\pi}{4}(n-1) \operatorname{sign} \lambda},
$$

valid in the space-time region (25) and

$$
(x-\xi) / t, y_{i} / t=O(1), \quad i=1, \ldots, n,
$$

on the paraboloid (12). Outside the paraboloid, the solution decays faster. This formula says that the localized initial condition (21) has evolved concentrating, asymptotically, on the paraboloid (12). 


\subsection{The nonlinear regime}

The approximate solution of $d K P_{n}$ in the nonlinear regime $t=O\left(\tau^{-1}\left(\epsilon^{-1}\right)\right)$, obtained matching equations (26) and (13), reads as follows:

$$
u(x, \vec{y}, t) \sim u_{n}^{a s}(x, \vec{y}, t) \equiv \begin{cases}t^{-\frac{n-1}{2}} \epsilon G\left(x+\frac{1}{4 t} \sum_{i=1}^{n-1} y_{i}^{2}-\frac{2 u t}{3-n}, \frac{\vec{y}}{2 t}\right), & n \neq 3, \\ t^{-1} \epsilon G\left(x+\frac{1}{4 t} \sum_{i=1}^{n-1} y_{i}^{2}-u t \ln t, \frac{\vec{y}}{2 t}\right), & n=3 .\end{cases}
$$

Since the term (ut) inside the first argument of function $G$, responsible for the wave breaking, is $O\left(t^{\frac{3-n}{2}}\right)$ for $n \neq 3$, and the analogous term $u t \ln t$ is $O(\ln t)$ for $n=3$, then these terms are large for $n=1,2,3$ and infinitesimal for $n \geq 4$. It follows that wave breaking takes place only for $n=1,2,3$; for $n \geq 4$ the solution (29) coincides with the linearized solution (26), and no breaking takes place. We remark that, in the integrable case $n=2$, one recovers the results obtained in [8] using the IST for vector fields. We also remark that, if the breaking regime (29) is known (measured), i.e., if function $G$ is known, the initial condition $\epsilon u_{0}(x, \vec{y})$ is uniquely reconstructed simply inverting (27).

It is possible to show that the error made approximating the solution of $d K P_{n}$ by (29) is given by $u=u_{n}^{a s}(x, \vec{y}, t)\left(1+O\left(t^{-1}\right)\right)$ for $n=2,3$.

Summarizing, the asymptotic solution (29) illustrates the following breaking picture for the $d K P_{n}$ equation (11), corresponding to localized and $O(\epsilon)$ initial data. If $n=1$ (the Riemann - Hopf case), waves break in the longtime regime $t=O\left(\epsilon^{-1}\right)$; if $n=2$, waves break in the longtime regime $t=O\left(\epsilon^{-2}\right)$, much later than in the $1+1$ dimensional case; also if $n=3$ small waves break, but at an exponentially large time scale: $t=O\left(e^{\frac{1}{\epsilon}}\right)$; at last, if $n \geq 4$, small and localized initial data do not break in the longtime regime. This result has a clear physical meaning: increasing the dimensionality of the transversal space, the number of diffraction channels of the wave increases, untill such diffraction, acting for a long time, is strong enough to prevent the gradient catastrophe of the small $n$ dimensional wave. It is a remarkable coincidence that small initial data break, in the longtime regime, only in $1+1,2+1$ and $3+1$ dimensions; i.e., only in physical space!

We end this section remarking that, if the initial condition is the Gaussian $u_{0}(x, \vec{y})=d_{n} \exp \left(-\frac{x^{2}+|\vec{y}|^{2}}{4}\right)$, where $d_{n}$ is a constant, then the above asymptotic solution can be written in terms of elementary or special functions, 
depending on $n$ :

$$
\begin{aligned}
& G(\xi, \vec{\eta})=\frac{d_{n}}{\sqrt{\pi}} \frac{1}{\left(1+|\vec{\eta}|^{2}\right)^{\frac{n+1}{4}}}\left[\cos \frac{\pi(n-1)}{4} \Gamma\left(\frac{n+1}{4}\right){ }_{1} F_{1}\left(\frac{n+1}{4}, \frac{1}{2} ;-\frac{Y^{2}}{4}\right)+\right. \\
& \left.\sin \frac{\pi(n-1)}{4} \Gamma\left(\frac{n+3}{4}\right) Y_{1} F_{1}\left(\frac{n+3}{4}, \frac{3}{2} ;-\frac{Y^{2}}{4}\right)\right] \\
& Y:=\frac{\xi}{\sqrt{1+|\vec{\eta}|^{2}}}
\end{aligned}
$$

where $\Gamma$ is the Euler Gamma function and ${ }_{1} F_{1}$ is the Kummer confluent hypergeometric function [21]. If, in particular, $n=2$ and $n=3$, with $d_{2}=\sqrt{2 \pi}$ and $d_{3}=2$, one obtains, respectively,

$$
\begin{aligned}
& G(\xi, \eta)=\left(1+\eta^{2}\right)^{-\frac{3}{4}}\left[\Gamma\left(\frac{3}{4}\right){ }_{1} F_{1}\left(\frac{3}{4}, \frac{1}{2} ;-\frac{Y^{2}}{4}\right)+\right. \\
& \left.Y \Gamma\left(\frac{5}{4}\right){ }_{1} F_{1}\left(\frac{5}{4}, \frac{3}{2} ;-\frac{Y^{2}}{4}\right)\right]
\end{aligned}
$$

(see Figure 1) and

$$
G\left(\xi, \eta_{1}, \eta_{2}\right)=\frac{\xi}{\left(1+\eta_{1}^{2}+\eta_{2}^{2}\right)^{\frac{3}{2}}} e^{-\frac{\xi^{2}}{4\left(1+\eta_{1}^{2}+\eta_{2}^{2}\right)}}
$$

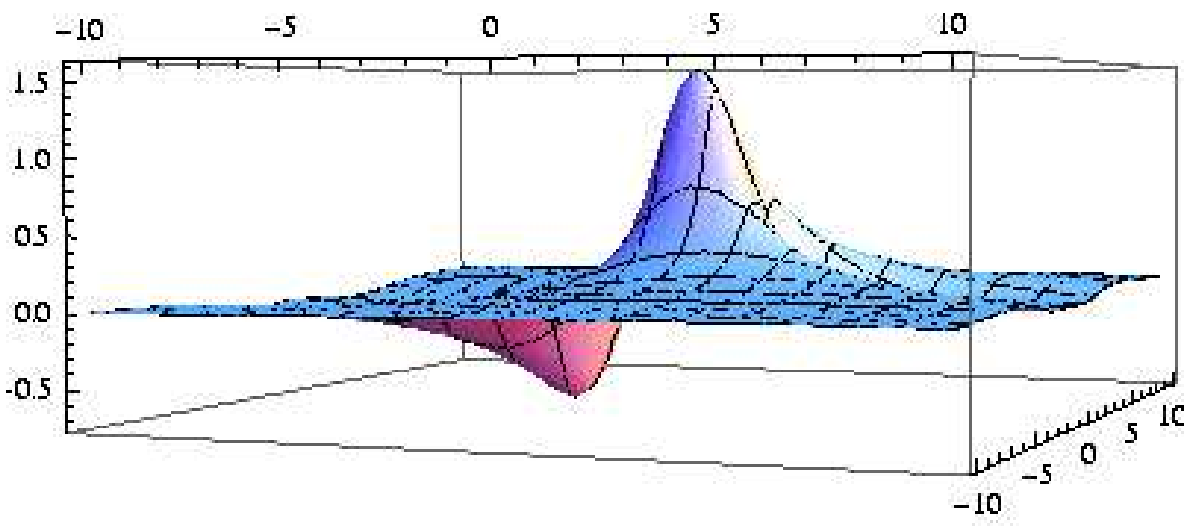

Figure 1. The plotting of function (31). 


\section{Geometric and analytic aspects of the wave breaking}

In this section we show how to derive, from the asymptotics (29), the analytic features of the wave breaking for $n=2,3$, in terms of the initial data, represented by function $G$ defined in (27). We first rewrite equations (29) in the more convenient form

$$
\begin{aligned}
& w \sim \epsilon G(\zeta, \vec{\eta}) \\
& \xi=\epsilon G(\zeta, \vec{\eta}) \tau+\zeta
\end{aligned}
$$

where

$$
w=\sqrt{t} u, \quad \tau=2 \sqrt{t}, \quad \xi=x+\frac{1}{4 t} y^{2}, \quad \eta=\frac{y}{2 t}
$$

for $n=2$, and

$$
w=t u, \quad \tau=\ln t, \quad \xi=x+\frac{1}{4 t}\left(y_{1}^{2}+y_{2}^{2}\right), \quad \vec{\eta}=\frac{\vec{y}}{2 t}
$$

for $n=3$, describing the evolution of an $n$-dimensional wave according to the $1+1$ dimensional Riemann - Hopf equation $w_{\tau}+w w_{\xi}=0$. In the following, we mainly concentrate on the case $n=3$; the case $n=2$, that can be easily recovered setting to zero all the partial derivatives of $G$ with respect to $\eta_{2}$ in the formulae of this section, has been discussed in detail in [8].

One solves the second of equations (33) with respect to the parameter $\zeta$, obtaining $\zeta(\xi, \vec{\eta}, \tau)$, and replaces it into the first, to obtain the solution $w \sim \epsilon G(\zeta(\xi, \vec{\eta}, \tau), \vec{\eta})$. The inversion of the second of equations (33) is possible iff its $\zeta$-derivative is different from zero. Therefore the singularity manifold (SM) of the solution is the $n$ - dimensional manifold characterized by the equation

$$
\mathcal{S}(\zeta, \vec{\eta}, \tau) \equiv 1+\epsilon G_{\zeta}(\zeta, \vec{\eta}) \tau=0 \quad \Rightarrow \quad \tau=-\frac{1}{\epsilon G_{\zeta}(\zeta, \vec{\eta})}
$$

Since

$$
\nabla_{(\xi, \vec{\eta})} w=\frac{\epsilon \nabla_{(\zeta, \vec{\eta})} G(\zeta, \vec{\eta})}{1+\epsilon G_{\zeta}(\zeta, \vec{\eta}) \tau}
$$

the slope of the localized wave becomes infinity (the so-called gradient catastrophe) on the SM, and the $n$-dimensional wave "breaks". 
Then the first breaking time $\tau_{b}$ and the corresponding characteristic parameters $\vec{\zeta}_{b}=\left(\zeta_{b}, \vec{\eta}_{b}\right)$ are defined by the global minimum of a function of $n$ variables:

$$
\tau_{b}=-\frac{1}{\epsilon G_{\zeta}\left(\vec{\zeta}_{b}\right)}=\text { global } \min \left(-\frac{1}{\epsilon G_{\zeta}(\zeta, \vec{\eta})}\right)>0
$$

and it is characterized, together with the condition $G_{\zeta}\left(\vec{\zeta}_{b}\right)<0$, by the condition that the symmetric quadratic form $<\underline{z}, \mathcal{H} \underline{z}>$ be positive $\forall \underline{z} \in \mathbb{R}^{n}$, where $\mathcal{H}$ is the Hessian matrix of function $G_{\zeta}(\zeta, \vec{\eta})$, evaluated at $\vec{\zeta}=\vec{\zeta}_{b}=\left(\zeta_{b}, \vec{\eta}_{b}\right)$.

The corresponding point at which the first wave breaking takes place is, from $(\underline{33}), \vec{\xi}_{b}=\left(\xi_{b}, \vec{\eta}_{b}\right) \in \mathbb{R}^{n}$, where:

$$
\xi_{b}=\zeta_{b}+\epsilon G\left(\vec{\zeta}_{b}\right) \tau_{b}
$$

Now we evaluate equations (33) and (36) near breaking, in the regime:

$$
\xi=\xi_{b}+\xi^{\prime}, \quad \vec{\eta}=\vec{\eta}_{b}+\vec{\eta}^{\prime}, \quad \tau=\tau_{b}+\tau^{\prime}, \quad \zeta=\zeta_{b}+\zeta^{\prime},
$$

where $\xi^{\prime}, \vec{\eta}^{\prime}, \tau^{\prime}, \zeta^{\prime}$ are small. Using (38) - (39), the second of equations (33) becomes, at the leading order, the following cubic equation in $\zeta^{\prime}$ :

$$
\zeta^{\prime 3}+a\left(\vec{\eta}^{\prime}\right) \zeta^{\prime 2}+b\left(\vec{\eta}^{\prime}, \tilde{\tau}\right) \zeta^{\prime}-\gamma X\left(\xi^{\prime}, \vec{\eta}^{\prime}, \tilde{\tau}\right)=0
$$

where

$$
\begin{aligned}
& a\left(\vec{\eta}^{\prime}\right)=\frac{3}{G_{\zeta \zeta \zeta}}\left(G_{\zeta \zeta \eta_{1}} \eta_{1}^{\prime}+G_{\zeta \zeta \eta_{2}} \eta_{2}^{\prime}\right), \\
& b\left(\vec{\eta}^{\prime}, \tilde{\tau}\right)=\frac{3}{G_{\zeta \zeta \zeta}}\left[2 G_{\zeta} \tilde{\tau}+G_{\zeta \eta_{1} \eta_{1}} \eta_{1}^{\prime 2}+2 G_{\zeta \eta_{1} \eta_{2}} \eta_{1}^{\prime} \eta_{2}^{\prime}+G_{\zeta \eta_{2} \eta_{2}} \eta_{2}^{\prime 2}\right], \\
& X\left(\xi^{\prime}, \vec{\eta}^{\prime}, \tilde{\tau}\right)=\xi^{\prime}-\epsilon G\left(\zeta_{b}, \vec{\eta}_{b}+\vec{\eta}^{\prime}\right) \tau^{\prime}-\epsilon\left[G\left(\zeta_{b}, \vec{\eta}_{b}+\vec{\eta}^{\prime}\right)-G\right] \tau_{b} \sim \\
& \xi^{\prime}+\left(\frac{G_{\eta_{1}}}{G_{\zeta}} \eta_{1}^{\prime}+\frac{G_{\eta_{2}}}{G_{\zeta}} \eta_{2}^{\prime}\right)-\frac{G}{\mid G_{\zeta}} \tilde{\tau}+\frac{1}{2 G_{\zeta}}\left(G_{\eta_{1} \eta_{1}} \eta_{1}^{\prime 2}+2 G_{\eta_{1} \eta_{2}} \eta_{1}^{\prime} \eta_{2}^{\prime}+\right. \\
& \left.G_{\eta_{2} \eta_{2}} \eta_{2}^{\prime 2}\right)-\frac{1}{\left|G_{\zeta}\right|}\left(G_{\eta_{1}} \eta_{1}^{\prime}+G_{\eta_{2}} \eta_{2}^{\prime}\right) \tilde{\tau}+\frac{1}{6 G_{\zeta}}\left(G_{\eta_{1} \eta_{1} \eta_{1}} \eta_{1}^{\prime 3}+\right. \\
& \left.3 G_{\eta_{1} \eta_{1} \eta_{2}} \eta_{1}^{\prime 2} \eta_{2}^{\prime}+3 G_{\eta_{1} \eta_{2} \eta_{2}} \eta_{1}^{\prime}{\eta_{2}^{\prime}}^{2}+G_{\eta_{2} \eta_{2} \eta_{2}} \eta_{2}^{\prime 3}\right), \quad \gamma=\frac{6\left|G_{\zeta}\right|}{G_{\zeta \zeta \zeta}}
\end{aligned}
$$

and

$$
\tilde{\tau} \equiv \frac{\tau^{\prime}}{\tau_{b}}=\frac{\tau-\tau_{b}}{\tau_{b}}
$$

corresponding to the maximal balance

$$
\left|\zeta^{\prime}\right|,\left|\eta_{1}^{\prime}\right|,\left|\eta_{2}^{\prime}\right|=O\left(|\tilde{\tau}|^{1 / 2}\right), \quad|X|=O\left(|\tilde{\tau}|^{3 / 2}\right) .
$$


In (42) and in the rest of this section, all partial derivatives of $G$ whose arguments are not indicated are meant to be evaluated at $\vec{\zeta}_{b}=\left(\zeta_{b}, \vec{\eta}_{b}\right)$.

The three roots of the cubic are given by the well-known Cardano-Tartaglia formula:

$$
\begin{aligned}
& \zeta_{1}^{\prime}\left(\xi^{\prime}, \vec{\eta}^{\prime}, \tilde{\tau}\right)=-\frac{a}{3}+\left(A_{+}\right)^{\frac{1}{3}}+\left(A_{-}\right)^{\frac{1}{3}} \\
& \zeta_{ \pm}^{\prime}\left(\xi^{\prime}, \vec{\eta}^{\prime}, \tilde{\tau}\right)=-\frac{a}{3}-\frac{1}{2}\left(\left(A_{+}\right)^{\frac{1}{3}}+\left(A_{-}\right)^{\frac{1}{3}}\right) \pm \frac{\sqrt{3}}{2} i\left(\left(A_{+}\right)^{\frac{1}{3}}-\left(A_{-}\right)^{\frac{1}{3}}\right),
\end{aligned}
$$

where

$$
A_{ \pm}=R \pm \sqrt{\Delta}
$$

and the discriminant $\Delta$ reads

$$
\Delta=R^{2}+Q^{3}
$$

with

$$
\begin{aligned}
& Q\left(\vec{\eta}^{\prime}, \tilde{\tau}\right)=\frac{3 b-a^{2}}{9}=-\frac{2\left|G_{\zeta}\right|}{G_{\zeta \zeta \zeta}} \tilde{\tau}+\frac{1}{G_{\zeta \zeta \zeta}^{2}}\left[\left(G_{\zeta \zeta \zeta} G_{\zeta \eta_{1} \eta_{1}}-G_{\zeta \zeta \eta_{1}}^{2}\right) \eta_{1}^{\prime 2}+\right. \\
& +2\left(G_{\zeta \zeta \zeta} G_{\zeta \eta_{1} \eta_{2}}-G_{\zeta \zeta \eta_{1}} G_{\zeta \zeta \eta_{2}}\right) \eta_{1}^{\prime} \eta_{2}^{\prime}+\left(G_{\zeta \zeta \zeta} G_{\zeta \eta_{2} \eta_{2}}-\right. \\
& \left.\left.G_{\zeta \zeta \eta_{2}}^{2}\right) \eta_{2}^{\prime 2}\right], \\
& R\left(\xi^{\prime}, \vec{\eta}^{\prime}, \tilde{\tau}\right)=\frac{\gamma}{2} X\left(\xi^{\prime}, \vec{\eta}^{\prime}, \tilde{\tau}\right)+\frac{a b}{18}+\frac{a}{3} Q\left(\vec{\eta}^{\prime}, \tilde{\tau}\right) .
\end{aligned}
$$

At the same order, function $\mathcal{S}$ in (36) becomes

$$
\begin{aligned}
& \mathcal{S}\left(\zeta^{\prime}, \vec{\eta}^{\prime}, \tilde{\tau}\right)=-\tilde{\tau}+\frac{1}{2\left|G_{\zeta}\right|}\left[G_{\zeta \zeta \zeta \zeta^{\prime 2}}+G_{\zeta \eta_{1} \eta_{1}} \eta_{1}^{\prime 2}+G_{\zeta \eta_{2} \eta_{2}} \eta_{2}^{\prime 2}+\right. \\
& \left.2 G_{\zeta \zeta \eta_{1}} \zeta^{\prime} \eta_{1}^{\prime}+2 G_{\zeta \zeta \eta_{2}} \zeta^{\prime} \eta_{2}^{\prime}+2 G_{\zeta \eta_{1} \eta_{2}} \eta_{1}^{\prime} \eta_{2}^{\prime}\right]= \\
& -\tilde{\tau}+\frac{1}{2\left|G_{\zeta}\right|}<\overrightarrow{\xi^{\prime}}, \mathcal{H} \vec{\xi}^{\prime}>, \quad \vec{\xi}^{\prime}=\left(\xi^{\prime}, \vec{\eta}^{\prime}\right) .
\end{aligned}
$$

Known $\zeta^{\prime}$ as function of $\left(\xi^{\prime}, \vec{\eta}^{\prime}, \tilde{\tau}\right)$ from the cubic (41), the solution $w$ and its gradient are then approximated, near breaking, by the formulae:

$$
\begin{aligned}
& w(\xi, \vec{\eta}, \tau) \sim \epsilon G\left(\zeta_{b}+\zeta^{\prime}, \vec{\eta}_{b}+\vec{\eta}^{\prime}\right) \\
& \nabla_{(\xi, \vec{\eta})} w \sim \epsilon \frac{\nabla_{\left(\zeta^{\prime}, \vec{\eta}^{\prime}\right)} G\left(\zeta_{b}+\zeta^{\prime}, \vec{\eta}_{b}+\vec{\eta}^{\prime}\right)}{\mathcal{S}\left(\zeta^{\prime}, \vec{\eta}^{\prime}, \tilde{\tau}\right)} .
\end{aligned}
$$

\subsubsection{Before breaking}

If $\tau<\tau_{b}(\tilde{\tau}<0)$, the coefficient $Q$ in (48) is strictly positive, due to the positivity of the Hessian quadratic form; then the discriminant $\Delta=R^{2}+Q^{3}$ 
is also strictly positive and only the root $\zeta_{1}^{\prime}$ is real. Correspondingly, the real solution $w$ is single valued and described by Cardano's formula (see Figure $2)$. In addition, function $\mathcal{S}$ in (49) is also strictly positive and $\nabla_{(\xi, \vec{\eta})} w$ is finite $\forall \xi, \vec{\eta}$.

To have a more explicit solution, we first restrict the asymptotic region to a narrower volume, so that the cubic (41) reduces to the linear equation $b \zeta^{\prime}=\gamma X$; then the solution exhibits a universal behaviour, coinciding with the following exact similarity solution of equation $w_{\tau}+w w_{\xi}=0$ :

$$
w \sim \frac{\xi-\xi_{b}+\left(G_{\eta_{1}} / G_{\zeta}\right)\left(\eta_{1}-\eta_{1 b}\right)+\left(G_{\eta_{2}} / G_{\zeta}\right)\left(\eta_{2}-\eta_{2 b}\right)}{\tau-\tau_{b}}=\frac{\vec{\nu} \cdot\left(\vec{\xi}-\vec{\xi}_{b}\right)}{\tau-\tau_{b}},
$$

describing the hyperplane tangent to the wave, where

$$
\vec{\nu}=\left(1, \frac{G_{\eta_{1}}}{G_{\zeta}}, \frac{G_{\eta_{2}}}{G_{\zeta}}\right)
$$

defines the breaking direction. In addition, in such a narrow volume:

$$
\nabla_{(\xi, \vec{\eta})} w \sim \frac{1}{\tau-\tau_{b}} \vec{\nu}
$$

We also look at the different balance: $\xi^{\prime}, \eta_{j}^{\prime}$ of the same order, and $\tilde{\tau} \leq O\left(\left|\eta_{j}^{\prime}\right|\right)$, suitable for taking the $\tau \uparrow \tau_{b}$ limit. In this case the cubic simplifies to $\zeta^{\prime 3} \sim \gamma X$ and

$$
\begin{aligned}
& w \sim \epsilon G\left(\zeta_{b}+\sqrt[3]{\gamma X\left(\xi^{\prime}, \vec{\eta}^{\prime}, \tilde{\tau}\right)}, \vec{\eta}_{b}+\vec{\eta}^{\prime}\right) \Rightarrow \\
& \nabla_{\xi, \vec{\eta}} w \sim \frac{1}{3} \sqrt[3]{\frac{6\left|G_{\zeta}\right|}{G_{\zeta \zeta \zeta}}} \frac{\epsilon \nabla_{\zeta, \vec{\eta}} G}{\sqrt[3]{\left(X\left(\xi^{\prime}, \vec{\eta}^{\prime}, \tilde{\tau}\right)\right)^{2}}} .
\end{aligned}
$$

\subsubsection{At breaking}

As $\tau \uparrow \tau_{b}$, the above tangent hyperplane (now tangent at the breaking point) has an infinite slope and equation $G_{\zeta} \xi^{\prime}+G_{\eta_{1}} \eta_{1}^{\prime}+G_{\eta_{2}} \eta_{2}^{\prime}=0$.

At the breaking time $\tau=\tau_{b}$ one can give an explicit description of the vertical inflection. If $\left|\xi^{\prime} / \eta_{1}^{\prime}\right|,\left|\xi^{\prime} / \eta_{2}^{\prime}\right|=O(1)$, the cubic (41) simplifies to $\zeta^{\prime 3}=\gamma X$ and equation (54) becomes

$$
w \sim \epsilon G\left(\zeta_{b}+\sqrt[3]{\gamma X_{b}}, \vec{\eta}_{b}+\vec{\eta}^{\prime}\right) \Rightarrow \nabla_{\xi, \vec{\eta}} w \sim \frac{\sqrt[3]{\gamma}}{3} \frac{\epsilon \nabla_{\zeta, \vec{\eta}} G}{\sqrt[3]{X_{b}^{2}}}
$$

where $X_{b}\left(\xi^{\prime}, \vec{\eta}^{\prime}\right) \equiv X\left(\xi^{\prime}, \vec{\eta}^{\prime}, 0\right)$ is defined in (42). We remark that $X_{b} \sim$ $\xi^{\prime}+\left(\frac{G_{\eta_{1}}}{G_{\zeta}} \eta_{1}^{\prime}+\frac{G_{\eta_{2}}}{G_{\zeta}} \eta_{2}^{\prime}\right)$, if $\xi^{\prime}=\alpha_{1} \eta_{1}^{\prime}+\alpha_{2} \eta_{2}^{\prime}, \alpha_{j} \neq-G_{\eta_{j}} / G_{\zeta}, j=1,2$, while $X_{b} \sim \frac{1}{2 G_{\zeta}}\left(G_{\eta_{1} \eta_{1}} \eta_{1}^{\prime 2}+2 G_{\eta_{1} \eta_{2}} \eta_{1}^{\prime} \eta_{2}^{\prime}+G_{\eta_{2} \eta_{2}} \eta_{2}^{\prime 2}\right)$ if $\alpha_{j}=-G_{\eta_{j}} / G_{\zeta}, j=1,2$. 
Equation (55) implies that, if $n=2$, all the derivatives of $w$ blow up at $\tau=\tau_{b}$, in the breaking point $\left(\xi_{b}, \eta_{b}\right)$, with the universal law $X_{b}^{-2 / 3}$, except the derivative along the transversal line $X_{b}\left(\xi^{\prime}, \eta^{\prime}\right)=0$, represented by the vector field $\hat{X}=X_{b \eta} \partial_{\xi}-\partial_{\eta}$, for which

$$
\left.\hat{X} w\right|_{\left(\xi_{b}, \eta_{b}\right)}=-\epsilon G_{\eta} .
$$

If $n=3$, the situation is similar: all derivatives of $w$ blow up at $\tau=\tau_{b}$, in the breaking point $\left(\xi_{b}, \vec{\eta}_{b}\right)$, with the universal law $X_{b}^{-2 / 3}$, except the derivatives along the transversal surface $X_{b}\left(\xi^{\prime}, \vec{\eta}^{\prime}\right)=0$, having as natural basis the vector fields $\hat{X}_{j}=X_{b \eta_{j}} \partial_{\xi}-\partial_{\eta_{j}}, j=1,2$, for which:

$$
\left.\hat{X}_{j} w\right|_{\left(\xi_{b}, \vec{\eta}_{b}\right)}=-\epsilon G_{\eta_{j}}, j=1,2 .
$$

\subsubsection{After breaking}

After breaking, the solution becomes three-valued in a compact region of the $(\xi, \vec{\eta})$ - space (see Figures 2, 3, 4), and does not describe any physics; nevertheless a detailed study of the multivalued region is important, in view of a proper regularization of the model.

If $\tau>\tau_{b}(\tilde{\tau}>0)$, in the regime (44), the SM equation $\mathcal{S}=0$ :

$$
\begin{aligned}
& 2\left|G_{\zeta}\right| \tilde{\tau}=G_{\zeta \zeta \zeta \zeta^{\prime 2}}+G_{\zeta \eta_{1} \eta_{1}} \eta_{1}^{\prime 2}+G_{\zeta \eta_{2} \eta_{2}}{\eta_{2}^{\prime}}^{2}+ \\
& 2 G_{\zeta \zeta \eta_{1}} \zeta^{\prime} \eta_{1}^{\prime}+2 G_{\zeta \zeta \eta_{2}} \zeta^{\prime} \eta_{2}^{\prime}+2 G_{\zeta \eta_{1} \eta_{2}} \eta_{1}^{\prime} \eta_{2}^{\prime}
\end{aligned}
$$

describes an ellipsoidal paraboloid in the $\left(\zeta^{\prime}, \vec{\eta}^{\prime}, \tilde{t}\right)$ space, with minimum at the breaking point $\left(\vec{\xi}_{b}, \tilde{\tau}_{b}\right)$.

Eliminating $\zeta^{\prime}$ from equations (58) and (41), one obtains the SM equation in space-time coordinates, coinciding with the $\Delta=0$ condition, where $\Delta, Q$ and $R$ are defined in (47) and (48).

For $n=2$, the SM is a closed curve with two cusps in the $(\xi, \eta)$ - plane at the points

$$
\pm \sqrt{\frac{2\left|G_{\zeta}\right| G_{\zeta \zeta \zeta}}{G_{\zeta \zeta \zeta} G_{\zeta \eta \eta}-G_{\zeta \zeta \eta}^{2}}}\left(\frac{G_{\eta}}{\left|G_{\zeta}\right|}, 1\right) \sqrt{\tilde{\tau}}
$$

(see Figure 3), corresponding to the conditions $Q=R=0$, on which the three real solutions of the cubic coincide. On the remaining part of the closed curve $\Delta=0$, two of the three real branches coincide: $w_{1} \sim \epsilon G\left(\zeta_{b}+\zeta_{1}^{\prime}, \vec{\eta}\right), w_{+}=$ $w_{-} \sim \epsilon G\left(\zeta_{b}+\zeta_{+}^{\prime}, \vec{\eta}\right)$. Outside the closed curve, $\Delta>0$ and the real solution 
$w$ is single valued; inside the closed surface $\Delta<0$ and the real solution $w$ is three valued. We remark that the transversal and longitudinal widths of such a closed curve are respectively $O\left(\tilde{\tau}^{\frac{1}{2}}\right)$ and $O\left(\tilde{\tau}^{\frac{3}{2}}\right)$. Therefore this curve develops, at $\tau=\tau_{b}$, from the breaking point $\left(\xi_{b}, \eta_{b}\right)$, with an infinite speed in the tranversal direction, and with zero speed in the longitudinal direction, recovering the results obtained in [8].

For $n=3$, the SM is a closed surface in the $(\xi, \vec{\eta})$ - space made of two surfaces having the same boundary: the transversal closed curve $Q=R=$ 0 (see Figure 4), on which the three real solutions of the cubic coincide. The $Q=0$ condition defines an ellipse in the $\left(\eta_{1}, \eta_{2}\right)$ plane with semi-axes $\sqrt{\frac{2\left|G_{\zeta}\right| G_{\zeta \zeta \zeta}}{\lambda_{ \pm}}} \sqrt{\tilde{\tau}}$, where

$$
\begin{aligned}
& \lambda_{ \pm}=\frac{a_{1}+a_{2} \pm \sqrt{\left(a_{1}-a_{2}\right)^{2}+4 c^{2}}}{2} \\
& a_{j}=G_{\zeta \zeta \zeta} G_{\zeta \eta_{j} \eta_{j}}-G_{\zeta \zeta \eta_{j}}^{2}, j=1,2, \quad c=G_{\zeta \zeta \zeta} G_{\zeta \eta_{1} \eta_{2}}-G_{\zeta \zeta \eta_{1}} G_{\zeta \zeta \eta_{2}} .
\end{aligned}
$$

As before, in the remaining part of the closed curve $\Delta=0$, two of the three real branches coincide. While the axes of the transversal closed curve $Q=$ $R=0$ are of $O\left(\tilde{\tau}^{\frac{1}{2}}\right)$, the thickness of the longitudinal region between the two surfaces is of $O\left(\tau^{\frac{3}{2}}\right)$. Therefore this closed surface develops, at $\tau=\tau_{b}$, from the breaking point $\left(\xi_{b}, \vec{\eta}_{b}\right)$, with an infinite speed in the tranversal directions, and with zero speed in the longitudinal direction. Intersecting this closed surface with any plane containing the $\xi$ - axis, one obtains a closed curve with two cusps as in Figure 3; therefore the closed curve $Q=R=0$ is made of all these cusps.

We end these considerations remarking that the similarity solution before breaking, the vertical inflection at breaking, and the compact threevalued region after breaking make clear the universal character of the gradient catastrophe of two- and three-dimensional waves evolving according to the Riemann-Hopf equation (see Figure 2).

Since the transformations (34), (35) are globally invertible, for $t \neq 0$ :

$$
\begin{gathered}
n=2: \quad u=\frac{1}{\sqrt{t}} w(\xi, \eta, \tau), \quad t=\frac{\tau^{2}}{4}, \quad x=\xi-\frac{\eta^{2} \tau^{2}}{4}, \quad y=\frac{\eta \tau^{2}}{2}, \\
n=3: \quad u=\frac{1}{t} w(\xi, \vec{\eta}, \tau), \quad t=e^{\tau}, \quad x=\xi-e^{\tau}\left(\eta_{1}^{2}+\eta_{2}^{2}\right), \quad \vec{y}=2 e^{\tau} \vec{\eta},
\end{gathered}
$$

all the above considerations are easily transfered to the $d K P_{n}$ case. In particular, small and localized initial data evolving according to the $d K P_{n}$ equa- 
tion (11) break, at $t_{b}=\tau_{b}^{2} / 4$ in the point $\left(x_{b}, y_{b}\right)=\left(\xi_{b}-\tau_{b}^{2} \eta_{b}^{2} / 4, \eta_{b} \tau_{b}^{2} / 2\right)$ of the parabolic wave front $x+y^{2} /\left(4 t_{b}\right)=\xi_{b}$ if $n=2$ [8], and at $t_{b}=e^{\tau_{b}}$ in the point $\left(x_{b}, \vec{y}_{b}\right)=\left(\xi_{b}-e^{\tau_{b}}\left|\vec{\eta}_{b}\right|^{2}, 2 e^{\tau_{b}} \vec{\eta}_{b}\right)$ of the paraboloidal wave front $x+\left(y_{1}^{2}+y_{2}^{2}\right) /\left(4 t_{b}\right)=\xi_{b}$ if $n=3$. In addition, all the previous considerations concerning the universal character of such a wave breaking: the similarity solution before breaking, the vertical inflection at breaking, and the compact three-valued space region after breaking, are transfered in a straightforward way to the $d K P_{n}$ equation, for $n=2,3$.

For instance, at $t=t_{b}$ and in the space region $\left|x^{\prime}+\eta_{1 b} y_{1}^{\prime}+\eta_{2 b} y_{2}^{\prime}\right|=$ $O\left(\left|y_{j}^{\prime}\right| /\left(2 t_{b}\right)\right)$ (the transformed of $\left.\left|\xi^{\prime}\right|=O\left(\left|\eta_{j}^{\prime}\right|\right)\right)$ equations (155) become

$$
\begin{aligned}
& u \sim \epsilon t_{b}^{-\frac{n-1}{2}} G\left(\zeta_{b}+\sqrt[3]{\gamma \tilde{X}_{b}\left(x^{\prime}, \vec{y}^{\prime}\right)}, \vec{\eta}_{b}+\frac{1}{2 t_{b}} \vec{y}^{\prime}\right) \quad \Rightarrow \\
& \nabla_{(x, \vec{y})} u \sim \epsilon t_{b}^{-\frac{n-1}{2}} \frac{\sqrt[3]{\gamma}}{3 \sqrt[3]{\tilde{X}_{b}^{2}}}\left(G_{\zeta}, G_{\zeta} \vec{\eta}_{b}+\frac{1}{2 t_{b}} \nabla_{\vec{\eta}} G\right),
\end{aligned}
$$

where $x^{\prime}=x-x_{b}, \vec{y}^{\prime}=\vec{y}-\vec{y}_{b}$ and $\tilde{X}_{b}\left(x^{\prime}, \vec{y}^{\prime}\right)=X_{b}\left(\xi^{\prime}, \vec{\eta}^{\prime}\right)$. Again, if $n=2$, all derivatives of $u$ at the breaking point $\left(x_{b}, y_{b}\right)$ blow up, except that along the transversal line $\tilde{X}_{b}\left(x^{\prime}, y^{\prime}\right)=0$, for which:

$$
\left.\left(\tilde{X}_{y} u_{x}-u_{y}\right)\right|_{\left(x_{b}, y_{b}\right)}=-\frac{\epsilon}{2 t_{b}^{3 / 2}} G_{\eta} .
$$

Analogously, if $n=3$, all derivatives of $u$ at the breaking point $\left(x_{b}, \vec{y}_{b}\right)$ blow up, except those along the transversal surface $\tilde{X}_{b}\left(x^{\prime}, \vec{y}^{\prime}\right)=0$, represented by the basis vector fields $\hat{\tilde{X}}_{j}=\tilde{X}_{b y_{j}} \partial_{x}-\partial_{y_{j}}, j=1,2$, for which:

$$
\left.\hat{\tilde{X}}_{j} u\right|_{\left(x_{b}, \vec{y}_{b}\right)}=-\frac{\epsilon}{2 t_{b}{ }^{2}} G_{\eta_{j}}, j=1,2 .
$$



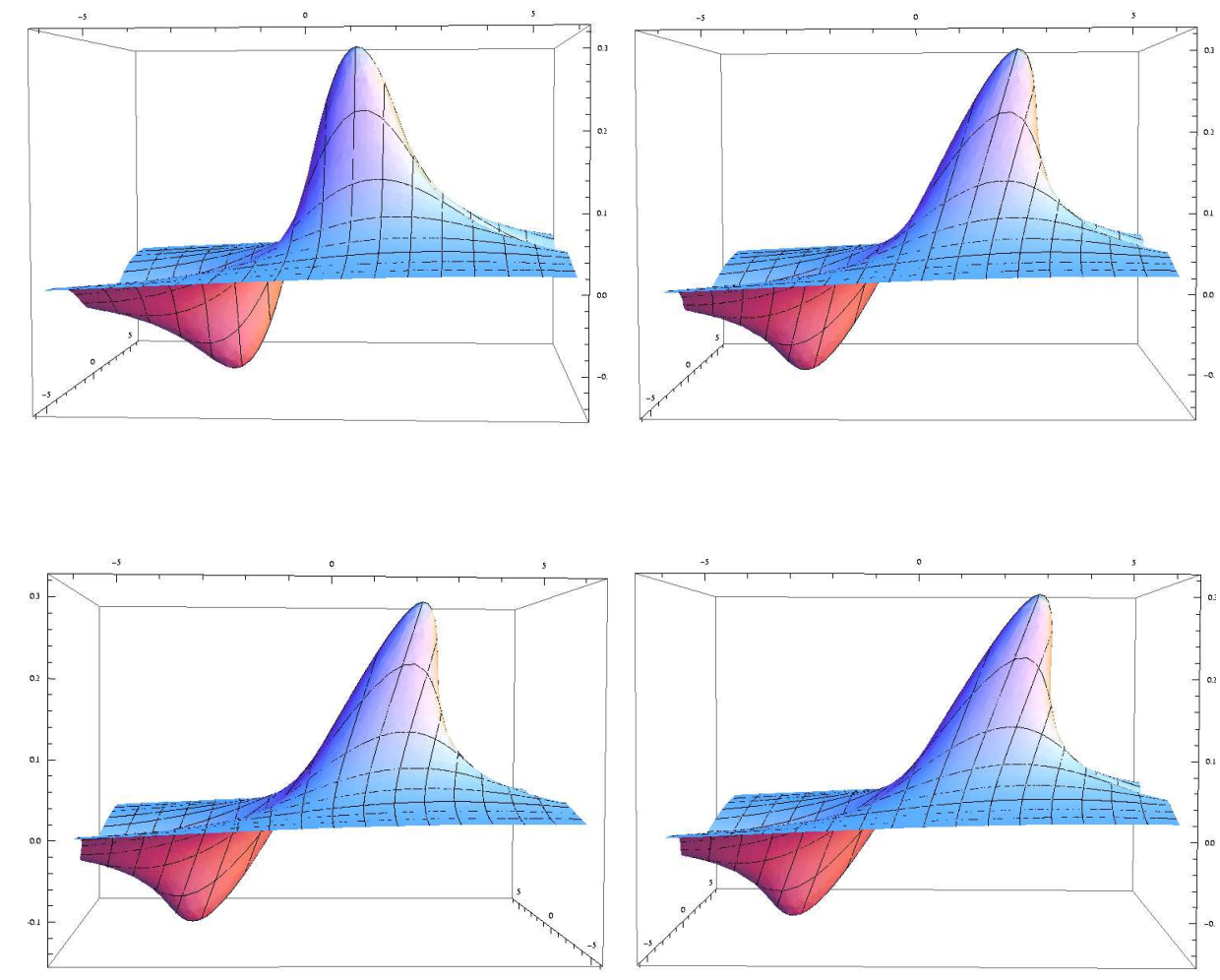

Figure 2. For $n=2, \epsilon=0.2$, and $G(\xi, \eta)$ given by (31), four consecutive snapshots, at $\tau=0, \tau=\tau_{b}-1, \tau=\tau_{b}$ and $\tau=\tau_{b}+1$, where $\tau_{b} \sim 6.57$, for the evolution described by (33). 

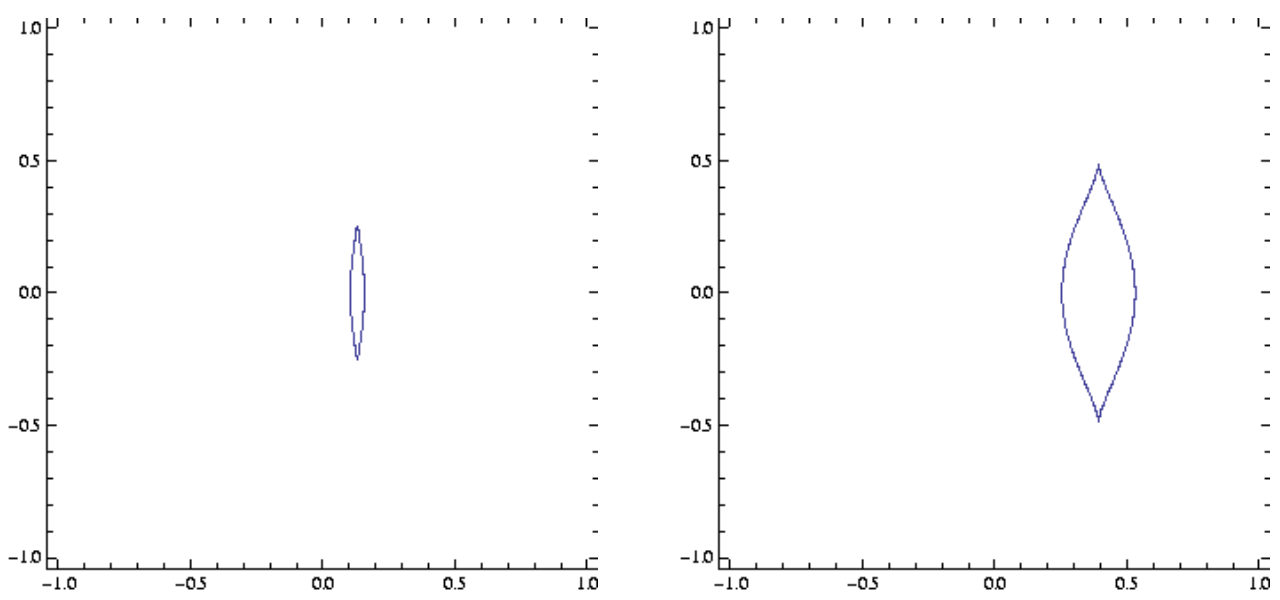

Fig. 3. For $n=2$ and $G(\zeta, \eta)$ given by (31), two consecutive snapshots, at $\tilde{\tau}=0.1$ and $\tilde{\tau}=0.3$, describing the evolution of the three-valued region, from the breaking point $\left(\xi_{b}, \eta_{b}\right) \sim(3.26,0)$ (the center of the figures). This region is delimited by a closed curve with two cusps.
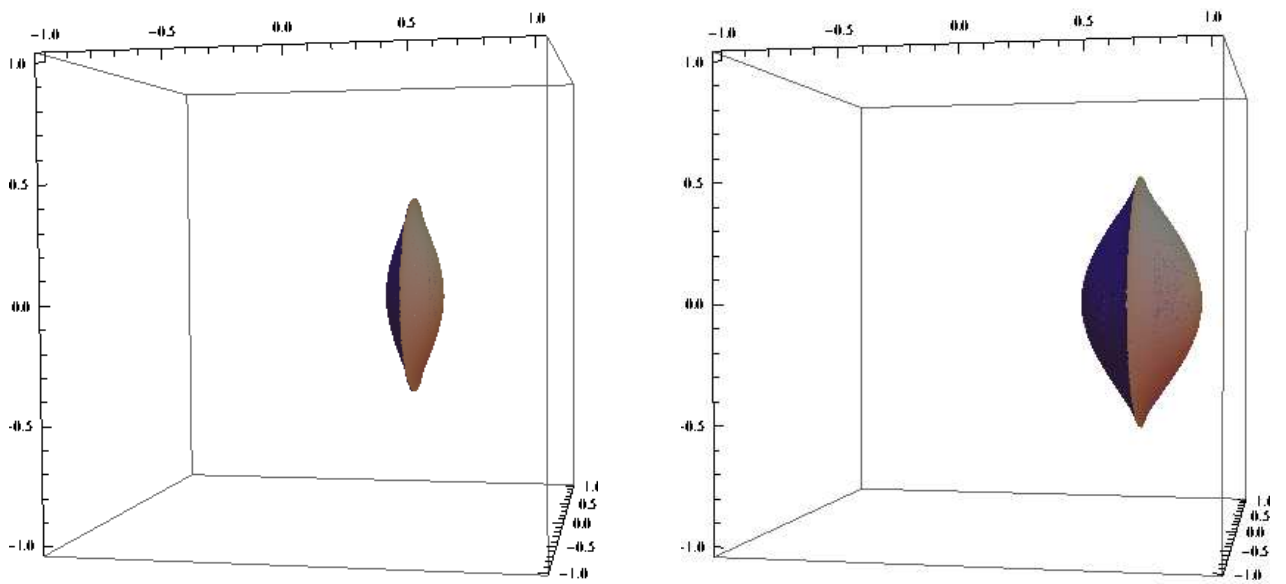

Figure 4. For $n=3$ and $G\left(\zeta, \eta_{1}, \eta_{2}\right)$ given by (32), two consecutive snapshots, at $\tilde{\tau}=0.3$ and $\tilde{\tau}=0.5$, describing the evolution of the compact three-valued region from the breaking point (the center of the figures). The closed surface delimiting the three-valued region is made of two surfaces with the same boundary, the closed curve $Q=R=0$. 


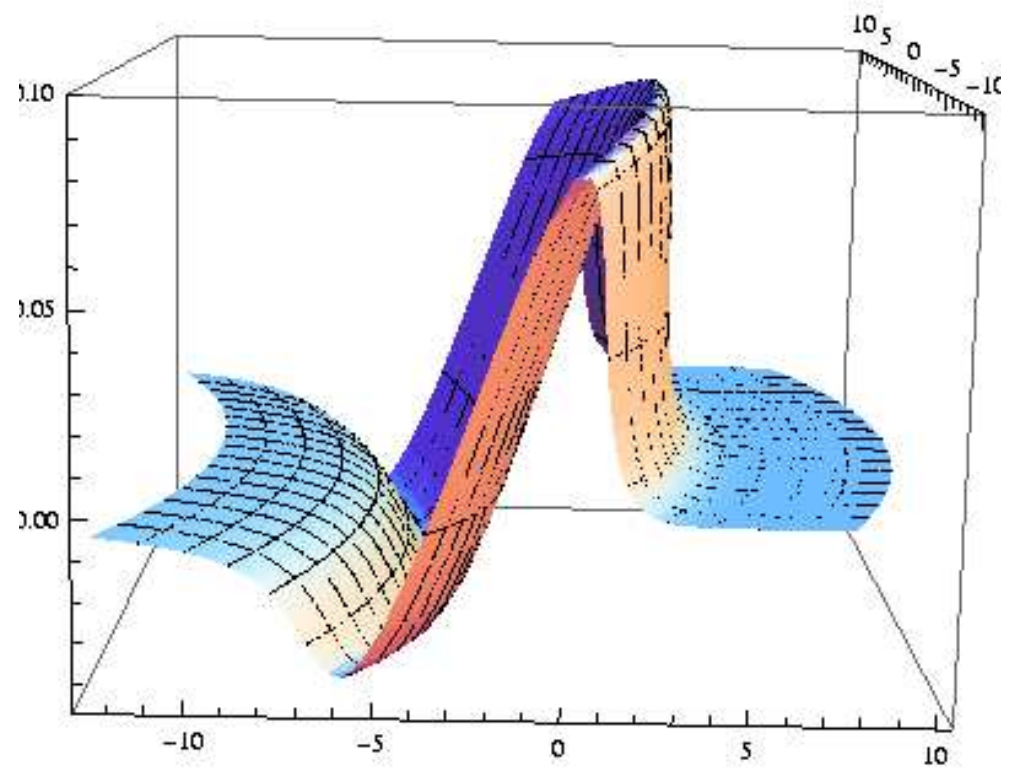

Fig. 5. For $n=2, \epsilon=0.2$ and $G(\zeta, \eta)$ given by (31), a detail of the parabolic wave front of $d K P_{2}$ at $t=t_{b}$, around the breaking point $\left(x_{b}, y_{b}\right)$.

Acknowledgements. This research has been supported by the RFBR grants 07-01-00446, 08-01-90104, and 09-01-92439, by the bilateral agreement between the Consortium Einstein and the RFBR, and by the bilateral agreement between the University of Roma "La Sapienza" and the Landau Institute for Theoretical Physics of the Russian Academy of Sciences.

\section{References}

[1] R. Timman, "Unsteady motion in transonic flow", Symposium Transsonicum, Aachen 1962. Ed. K. Oswatitsch, Springer 394-401.

[2] E. A. Zobolotskaya and R. V. Kokhlov, "Quasi - plane waves in the nonlinear acoustics of confined beams", Sov. Phys. Acoust. 15, n. 1 (1969) 35-40. 
[3] B. B. Kadomtsev and V. I. Petviashvili, "On the stability of solitary waves in weakly dispersive media", Sov. Phys. Dokl., 15, 539-541 (1970).

[4] V. E. Zakharov, S. V. Manakov, S. P. Novikov and L. P. Pitaevsky, Theory of solitons, Plenum Press, New York, 1984.

[5] M. J. Ablowitz and P. A. Clarkson, Solitons, nonlinear evolution equations and Inverse Scattering, London Math. Society Lecture Note Series, vol. 194, Cambridge University Press, Cambridge (1991).

[6] R. K. Dodd, J. C. Eilbeck, J. D. Gibbon, H. C. Morris, Solitons and nonlinear wave equations, Academic Press, 1982.

[7] J. B. Witham, Linear and nonlinear waves, J. Wiley and sons, New York, 1974 .

[8] S. V. Manakov and P. M. Santini: "On the solutions of the dKP equation: the nonlinear Riemann-Hilbert problem, longtime behaviour, implicit solutions and wave breaking"; J. Phys. A: Math. Theor. 41 (2008) 055204 (23pp).

[9] Y. Kodama and J. Gibbons, "Integrability of the dispersionless KP hierarchy", Proc. 4th Workshop on Nonlinear and Turbulent Processes in Physics, World Scientific, Singapore 1990.

[10] K. Takasaki, "Quasi-classical limit of BKP hierarchy and $W$-infinity symmetries". Lett. Math. Phys. 28 (1993), no. 3, 177-185.

[11] V. E. Zakharov, "Dispersionless limit of integrable systems in $2+1$ dimensions", in Singular Limits of Dispersive Waves, edited by N. M. Ercolani et al., Plenum Press, New York, 1994.

[12] I. M. Krichever, "The $\tau$-function of the universal Witham hierarchy, matrix models and topological field theories", Comm. Pure Appl. Math. 47, 437-475 (1994).

[13] K. Takasaki and T. Takebe, "Integrable hierarchies and dispersionless limit", Rev. Math. Phys. 7, 743-808 (1995).

[14] E. V. Ferapontov and K. R. Khusnutdinova: "On integrability of $(2+1)$ dimensional quasilinear systems", Comm. Math. Phys. 248 (2004) 187206. 
[15] S. V. Manakov and P. M. Santini: "The Cauchy problem on the plane for the dispersionless Kadomtsev-Petviashvili equation"; JETP Letters, 83, No 10, 462-466 (2006). http://arXiv:nlin.SI/0604016.

[16] S. V. Manakov and P. M. Santini: "Inverse scattering problem for vector fields and the Cauchy problem for the heavenly equation", Physics Letters A 359 (2006) 613-619.

[17] S. V. Manakov and P. M. Santini: "A hierarchy of integrable PDEs in $2+1$ dimensions associated with 1 - dimensional vector fields"; Theor. Math. Phys. 152(1), 1004-1011 (2007).

[18] S. V. Manakov and P. M. Santini: "The dispersionless 2D Toda equation: dressing, Cauchy problem, longtime behaviour, implicit solutions and wave breaking", J. Phys. A: Math. Theor. 42 (2009) 095203 (16pp).

[19] S. V. Manakov and P. M. Santini: "Solvable vector nonlinear Riemann problems, exact implicit solutions of dispersionless PDEs and wave breaking", arXiv:1011.2619. (J. Phys. A: Math. Theor., submitted to).

[20] V. G. Kamensky and S. V. Manakov, "Formation of instability regions from unstable states in nonlinear systems with dissipation". Nonlinear evolutions (Balaruc-les-Bains, 1987), 531-535, World Sci. Publ., Teaneck, NJ, 1988.

[21] Abramowitz and Stegun: Handbook of Mathematical Functions with Formulas, Graphs, and Mathematical Tables, Dover Publications, New York (1972). 